\section{(2) \\ BRAZIILIAN JOURNAL \\ OF MEDICAL AND BIOLOGICAL RESEARCH}

www.bjournal.com.br
ISSN 0100-879X

Volume 45 (5) 376-472 May 2012

BIOMEDICAL SCIENCES

AND

CLINICAL INVESTIGATION

Braz J Med Biol Res, May 2012, Volume 45(5) 411-416

doi: $10.1590 / \mathrm{S} 0100-879 X 2012007500034$

Negative correlation between phospholipase and esterase activity produced by Fusarium isolates

K. Ishida, D.S. Alviano, B.G. Silva, C.R. Guerra, A.S. Costa, M. Nucci, C.S. Alviano and S. Rozental

The Brazilian Journal of Medical and Biological Research is partially financed by

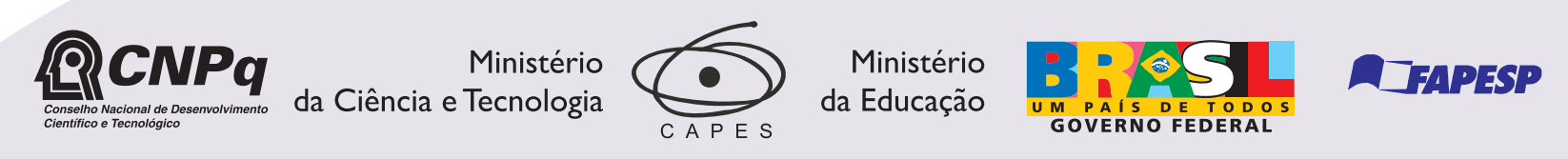

Institutional Sponsors

scielo
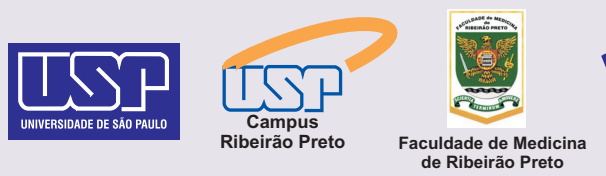

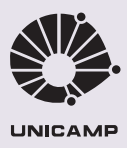

UNICAMP $\oplus$ SHIMADZU

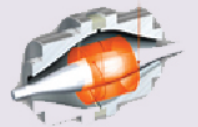

1 D. Associaçäo Explore High - Performance MS Orbitrap Technology In Proteomics \& Metabolomics analitica $\underset{\text { analiticaweb.com.br }}{\text { Thermo }}$ S I E N I FIC 


\title{
Negative correlation between phospholipase and esterase activity produced by Fusarium isolates
}

\author{
K. Ishida ${ }^{1}$, D.S. Alviano ${ }^{3}$, B.G. Silva ${ }^{1,3}$, C.R. Guerra ${ }^{1}$, A.S. Costa ${ }^{1}$, \\ M. Nucci ${ }^{2}$, C.S. Alviano ${ }^{3}$ and S. Rozental ${ }^{1}$ \\ ${ }^{1}$ Laboratório de Biologia Celular de Fungos, Instituto de Biofísica Carlos Chagas Filho, \\ Universidade Federal do Rio de Janeiro, Rio de Janeiro, RJ, Brasil \\ 2Departamento de Clínica Médica, Serviço de Hematologia, Hospital Universitário Clementino Fraga Filho, \\ Universidade Federal do Rio de Janeiro, Rio de Janeiro, RJ, Brasil \\ ${ }^{3}$ Departamento de Microbiologia Geral, Instituto de Microbiologia Prof. Paulo de Góes, \\ Universidade Federal do Rio de Janeiro, Rio de Janeiro, RJ, Brasil
}

\begin{abstract}
Fusarium species have emerged as one of the more outstanding groups of clinically important filamentous fungi, causing localized and life-threatening invasive infections with high morbidity and mortality. The ability to produce different types of hydrolytic enzymes is thought to be an important virulence mechanism of fungal pathogens and could be associated with the environment of the microorganism. Here, we have measured the production of two distinct lipolytic enzymes, phospholipase and esterase, by sixteen Fusarium isolates recovered from the hospital environment, immunocompromised patients' blood cultures, foot interdigital space scrapings from immunocompromised patients, and foot interdigital space scrapings from immunocompetent patients (4 isolates each). Fourteen of these 16 isolates were identified as Fusarium solani species complex (FSSC) and two were identified as F. oxysporum species complex (FOSC). Some relevant genus characteristics were visualized by light and electron microscopy such as curved and multicelled macroconidia with 3 or 4 septa, microconidia, phialides, and abundant chlamydospores. All Fusarium isolates were able to produce esterase and phospholipase under the experimental conditions. However, a negative correlation was observed between these two enzymes, indicating that a Fusarium isolate with high phospholipase activity has low esterase activity and vice versa. In addition, Fusarium isolated from clinical material produced more phospholipases, while environmental strains produced more esterases. These observations may be correlated with the different types of substrates that these fungi need to degrade during their nutrition processes.
\end{abstract}

Key words: Fusarium; Hospital isolates; Phospholipase; Esterase

\section{Introduction}

Fusarium species are important pathogens causing invasive fungal disease in severely immunocompromised patients, especially those with hematologic malignancies (1). More than 50 species of Fusarium have been identified, including plant and animal pathogens, but only few species are related to human disease. The more frequent species causing human disease are Fusarium solani, F. oxysporum and F. moniliforme (1). Traditionally, the identification of Fusarium species has been performed by observation of the macro- and micromorphologic characteristics of the mycelium and reproductive structures such as colony pigmentation, banana-shaped or oval conidial morphology, and the presence of multicellular macroconidia with a foot cell at the base. However, these methods are laborious, time-consuming and require a qualified professional. More recently, molecular techniques have replaced morphological ones and are considered to be gold standard procedures for fungal identification (2).

The major portal of entry for Fusarium spp in human disease is the inhalation of air-spread aerosols of Fusarium conidia, followed by skin tissue breakdown. The hospital water system may serve as a reservoir for Fusarium spp

Correspondence: S. Rozental, Laboratório de Biologia Celular de Fungos, Instituto de Biofísica Carlos Chagas Filho, UFRJ, Av. Carlos Chagas Filho, 373, Bloco C, Sala C0-026, 21941-902 Rio de Janeiro, RJ, Brasil. Fax: +55-21-2280-8193.

E-mail: rozental@biof.ufrj.br

Received November 28, 2011. Accepted February 28, 2012. Available online March 16, 2012. Published May 7, 2012. 
and showering may be an efficient mechanism for the airborne dispersion of fungal conidia and its transmission to immunocompromised hosts in the hospital environment (3).

The clinical forms of fusariosis depend largely on the immune status of the host and the portal of entry of the infection. Keratitis and onychomycosis are the most frequent diseases in immunocompetent individuals, whereas disseminated (and frequently fatal) disease predominates in severely immunocompromised patients (1). Several fungal virulence factors are associated with the pathogenesis of fusariosis, such as the ability to adhere to prosthetic material, secretion of mycotoxins (trichothecenes), which suppress humoral and cellular immunity and may cause tissue breakdown, and production/secretion of extracellular enzymes including proteases, collagenases, phospholipases, and esterases (4).

In general, the lipases, including phospholipases and esterases, are enzymes that hydrolyze phospholipids to fatty acids. Lipases have an important function in fungal infection as they hydrolyze fat and provide fatty acid residues for fungal nutrition, mainly in the subcutis and cuts (5). Depending on the different and specific ester bonds cleaved, these enzymes have been classified into phospholipases A, B, C, and D. Phospholipase production is associated with host cell-membrane damage, which can promote cell damage and/or expose receptors to facilitate adherence and fungal invasion (6). Esterase (or monoacylglycerol lipase) hydrolyzes monoacylglycerol with a long-chain fatty acid $\left(\mathrm{C}_{12}\right.$ or more).

The aim of the present study was to determine the production of extracellular enzymes, such as phospholipase and esterase, by Fusarium species isolates recovered from biological material from immunocompetent and immunocompromised patients, and from the hospital environment.

\section{Material and Methods}

\section{Fungal isolates}

We selected 16 Fusarium isolates recovered from the wall and water of the bathroom of the intensive care unit (IA1, IA-2, IA-3, and IA-4), immunocompromised patients' blood cultures $(\mathrm{H}-1, \mathrm{H}-2, \mathrm{H}-3$, and $\mathrm{H}-4)$, foot interdigital space scrapings from immunocompromised patients (RTMO-1, RTMO-2, RTMO-3, and RTMO-4), and foot interdigital space scrapings from immunocompetent patients (RIC-1, RIC-2, RIC-3, and RIC-4). Fourteen of these 16 isolates were identified as F. solanispecies complex (FSSC) and two were identified as F. oxysporum species complex (FOSC) by sequencing as previously described (2) and reported elsewhere (7). Fusarium sp isolates were cultivated on Sabouraud dextrose agar (SDA) plates for 10 days at $28^{\circ} \mathrm{C}$ and subcultures were then taken at the same time and under the same growth conditions.

\section{Morphological analysis}

Light microscopy. Mycelial structures of each isolate were carefully examined in the preparations obtained from microcultures inoculated onto SDA medium between glass slides and coverslips. Briefly, this technique is performed using a sterile $15-\mathrm{cm}$ diameter Petri dish whose bottom is covered with filter paper or cotton mesh, and 2 glass slides are placed onto a U-shaped glass rod. SDA medium was cut with a sterile scalpel blade into $1-\mathrm{cm}^{2}$ squares, which were placed on each microculture chamber slide. Each fungal colony was inoculated onto the 4 sides of each culture medium square and a sterile coverslip was placed over the medium. The microculture chamber was maintained at $28^{\circ} \mathrm{C}$ for 7 to 10 days under humid conditions for the observation of colony growth and conidiation. The vegetative and reproductive mycelia of Fusarium sp adhered to the coverslips were stained with lactophenol cotton blue, dripped onto the glass slides and examined with light microscope (Axiostar plus, Zeiss, Germany).

Scanning electron microscopy. Fusarium $\mathrm{sp}$ isolates obtained by microculture as described previously were fixed in a solution of $2.5 \%$ glutaraldehyde and $4 \%$ freshly prepared formaldehyde in $0.1 \mathrm{M}$ cacodylate buffer, $\mathrm{pH} 7.2$, for $2 \mathrm{~h}$ at room temperature. After fixation, the isolates were post-fixed for $2 \mathrm{~h}$ in $1 \%$ osmium tetroxide containing $1.25 \%$ potassium ferrocyanide and $5 \mathrm{mM} \mathrm{CaCl}_{2}$ in $0.1 \mathrm{M}$ cacodylate buffer, $\mathrm{pH} 7.2$, dehydrated in ethanol, critical-point dried in $\mathrm{CO}_{2}$, coated with gold, and then observed with a JEOL JSM-5310 (Japan) scanning electron microscope.

\section{Phospholipase assay}

Phospholipase production was determined using egg yolk agar plates (SDA medium supplemented with $1 \mathrm{M}$ $\mathrm{NaCl}, 5 \mathrm{mM} \mathrm{CaCl}_{2}$, and $8 \%$ sterile egg yolk emulsion) as described by Price et al. (8). In this method, egg yolk digested by phospholipase produces precipitation around fungal colonies. Ten microliters $\left(1 \times 10^{7}\right.$ conidia $)$ of a suspension of each strain was placed in the center of the egg yolk agar plate (9-cm diameter Petri dishes). After incubation at $37^{\circ} \mathrm{C}$ for 15 and 30 days, the colony diameter (a) and the diameter of the colony plus the precipitation zone (b) were measured with a digital pachymeter.

\section{Esterase assay}

Esterase production by Fusarium was assayed using SDA medium, consisting of $10 \mathrm{~g}$ peptone, $5 \mathrm{~g} \mathrm{NaCl}, 0.1 \mathrm{~g}$ $\mathrm{CaCl}_{2}, 15 \mathrm{~g}$ agar, and $1000 \mathrm{~mL}$ distilled water, with $\mathrm{pH}$ adjusted to $\mathrm{pH} 6.5$ (9). The medium was autoclaved, cooled to about $50^{\circ} \mathrm{C}$, and $5 \mathrm{~mL}$ autoclaved Tween 80 (Sigma, USA) was added. The suspension of $1 \times 10^{7}$ conidia $(10 \mu \mathrm{L})$ was inoculated and plates were incubated at $37^{\circ} \mathrm{C}$ up to 30 days. The colony diameter (a) and the diameter of the colony plus the precipitation zone (b) were measured with a digital pachymeter. 


\section{Enzymatic score and statistical analysis}

Phospholipase and esterase activities are reported as $\mathrm{Pz}$ value (a/b) as described by Price et al. (8). According to this definition, low $\mathrm{Pz}$ values indicate high phospholipase or esterase production and, conversely, high $\mathrm{Pz}$ values indicate low enzymatic production. The Pz value was scored into four categories: high $\mathrm{Pz}$ values of 1.0 were scored as negative enzymatic production, $\mathrm{Pz}$ values between 0.999 and 0.700 as low enzymatic production (+), Pz values between 0.699 and 0.400 as moderate enzymatic production (++), and $\mathrm{Pz}$ values between 0.399 and 0.100 as high enzymatic production (+++). All experiments were repeated at least three times and all systems were performed in triplicate.

Data were analyzed statistically using the GraphPad
Prism program version 4.0 and a $P$ value $<0.05$ was considered to be significant. The Pearson test was performed to correlate phospholipase and esterase activities and the Student $t$-test to compare the enzyme production by the fungal isolate group (IA, H, RTMO, and RIC).

\section{Results and Discussion}

Typical morphological characteristics of the F. solani and F. oxysporum species complex (FSSC and FOSC, respectively) were observed independently of the site of fungal isolate, as visualized by light and scanning electron microscopy (Figure 1). The 14 FSSC isolates presented characteristic structures of this group such as curved and
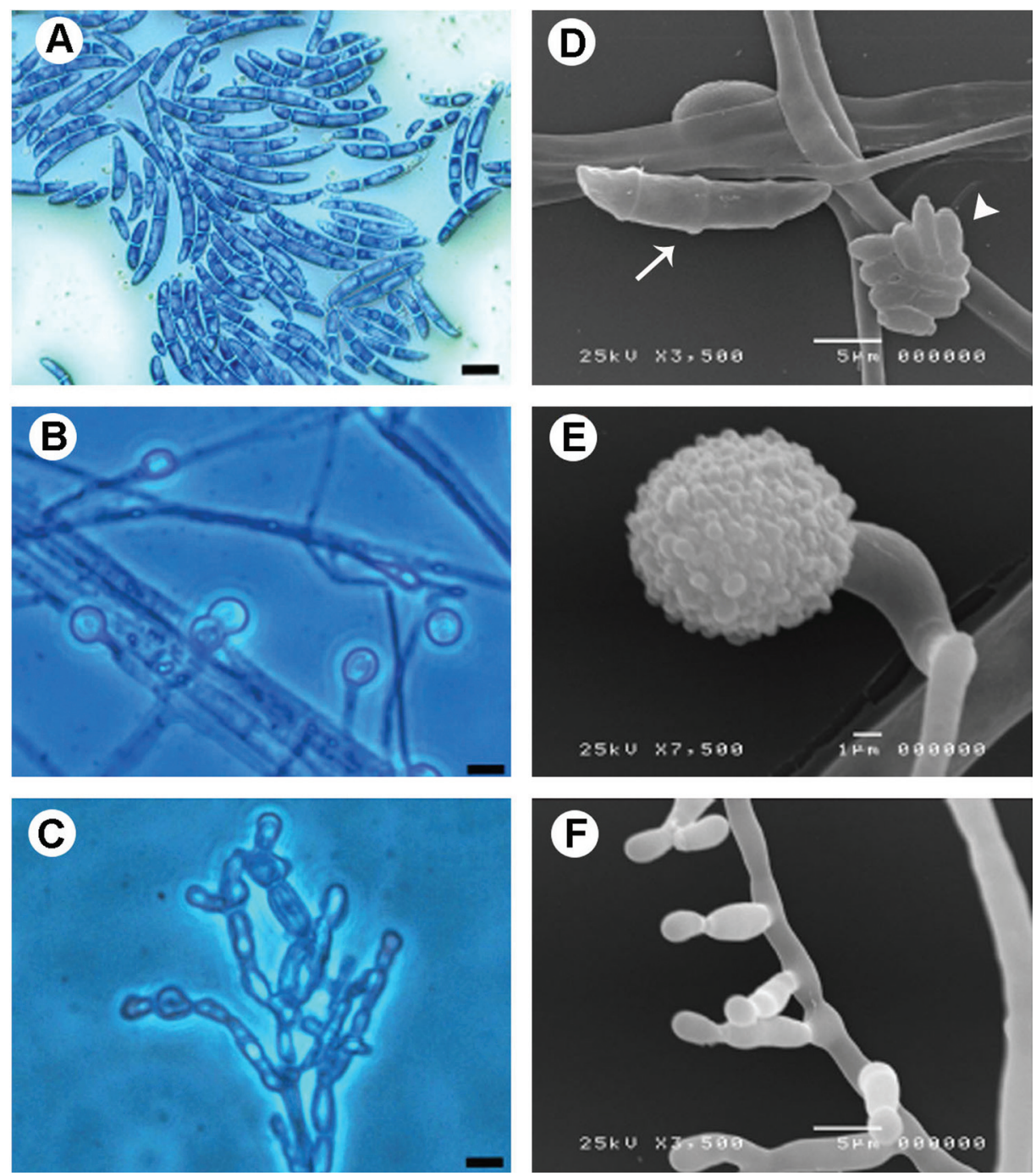

Figure 1. Light $(A-C)$ and scanning electron microscopy micrographs $(D-F)$ of Fusarium spp reproductive structures. Fusarium oxysporum $(A)$ and $F$. solani species complex $(D$, white arrow) macroconidia. $F$. solani species complex microconidia can also be observed in $D$ (white arrowhead). $F$. oxysporum $(B)$ and $F$. solani $(E)$ species complex chlamydospores. $F$. oxysporum $(F)$ and $F$. solani (C) species complex phialides. Scale bars: $20 \mu \mathrm{m}(A-C), 5 \mu \mathrm{m}(D$ and $F)$ and $1 \mu \mathrm{m}(E)$. 
multicelled macroconidia with 3 or 4 septa (Figure 1D, white arrow), microconidia (Figure 1D, white arrowhead), lengthy phialides (Figure 1C) and abundant chlamydospores (Figure $1 \mathrm{E}$ ). The two FOSC isolates (both recovered from the intensive care bathroom) presented curved and multicelled macroconidia with 1-4 septa (Figure 1A), chlamydospores (Figure 1B) and short phialides (Figure 1F).

Although the clinical manifestations and outcome of fusariosis depend largely on the immune status of the host, the virulence attributes expressed by the fungus enhance the infective process (1). The fungal adhesion to host tissue is an extremely important step for establishing the infection and tissue invasion, by digesting and destroying cell membranes and/or extracellular matrix components (10). The activity of hydrolytic enzymes, including proteases, lipases (phospholipases and esterases) and hemolysins, is associated with adhesion, cell damage, tissue invasion, and blood dissemination in several infections caused by bacteria and fungi (10).

Herein, we evaluated the extracellular esterase (Figure $2 \mathrm{~B}$ ) and phospholipase (Figure $2 \mathrm{C}$ ) production by the 16 Fusarium isolates cultured on modified Sabouraud medium for 15 and 30 days. These assays, which detect phospholipase and esterase activities by measurement of the zone precipitation around colonies, have been largely employed to study enzyme production by various bacteria and fungi $(8,11,12)$. In the present study, all isolates were able to secrete esterase and phospholipase enzymes (Figure $2 \mathrm{~A}-\mathrm{C}$ and Table 1). The phospholipase and esterase activities were time-dependent, increasing with culture growth time (Table 1). After 30 days of culture, most of the isolates showed moderate phospholipase and/or esterase activity. Interestingly, we observed that three Fusarium isolates from the hospital environment (IA-1, IA-2, and IA-3) presented the highest esterase activity (lowest $\mathrm{Pz}$ values of $0.3-0.37$ ) (Table 1 and Figure $2 \mathrm{E}$ ), whereas the isolates scraped from the interdigital spaces presented the highest phospholipase activities (Table 1). It is important to remember that low $\mathrm{Pz}$ values mean high phospholipase or esterase production and, conversely, high $\mathrm{Pz}$ values indicate low enzymatic production.

Additionally, we observed a negative correlation ( $r=$ $-0.43, \mathrm{P}<0.05$, Pearson test) between the two lipases studied (phospholipases and esterases; Figure 2D), indicating
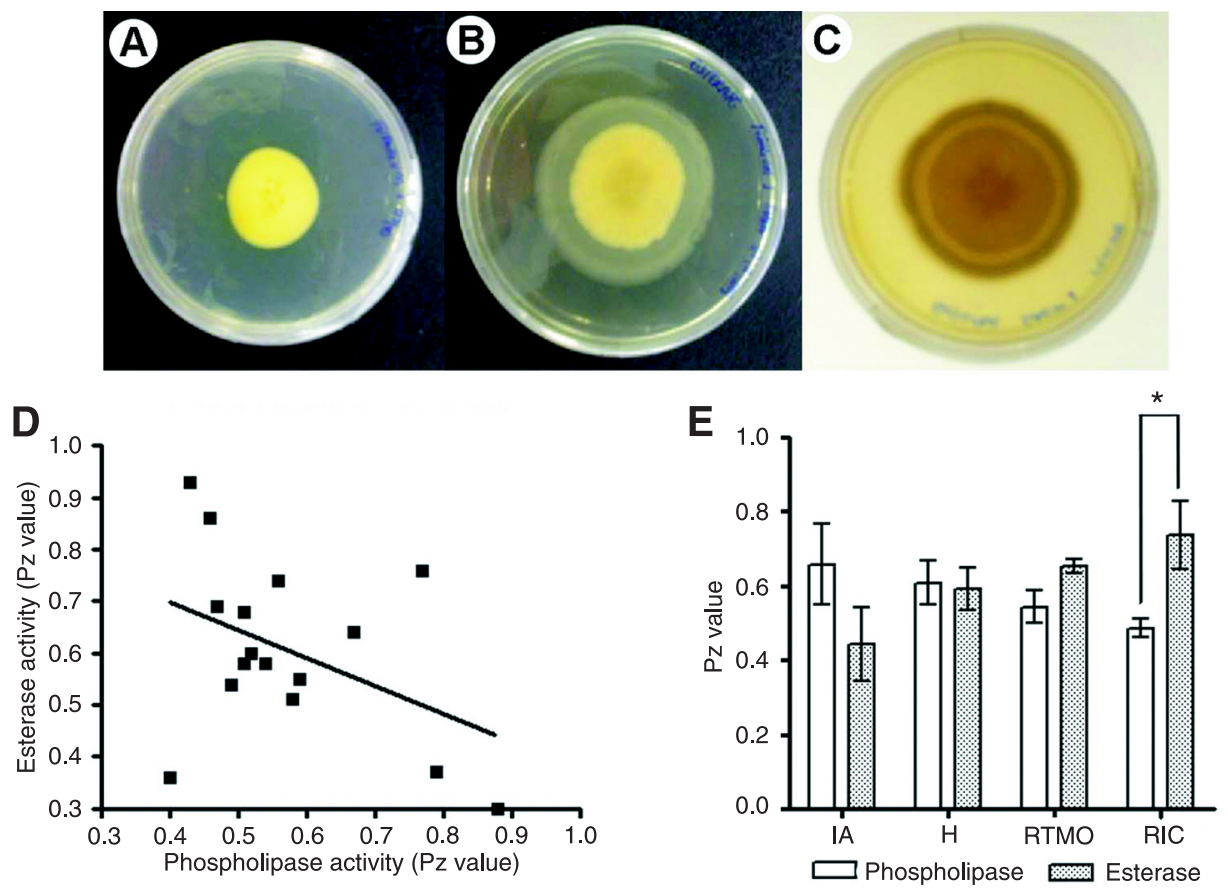

Figure 2. Phospholipase and esterase activities of Fusarium spp isolates. A, Fusarium sp grown on Sabouraud dextrose agar (SDA, control); B, Fusarium sp grown on SDA supplemented with $0.1 \%$ Tween 80 - esterase production; C, Fusarium sp on SDA supplemented with $2 \%$ egg yolk - phospholipase production. D, Negative correlation between phospholipase and esterase $\mathrm{Pz}$ values extracted from Table 1 ( $r=-0.43, P<0.05$, Pearson test); E, phospholipase and esterase activities by the following fungal isolate groups: hospital environment (IA), blood cultures $(\mathrm{H})$ from immunocompromised patients, foot interdigital space scrapings from immunocompromised patients (RTMO), and foot interdigital space scrapings from immunocompetent patients (RIC). ${ }^{*} \mathrm{P}<0.05$ (Student $t$-test). 
that Fusarium isolates with high phospholipase activity have a low esterase production and vice versa. The production of enzymes was also analyzed according to fungal group (IA, $\mathrm{H}, \mathrm{RTMO}$, and RIC) and compared between them (Figure 2E). We observed that Fusarium isolates from clinical material produced more phospholipase, an enzyme that degrades complex lipids such as phospholipids present in tissues. In contrast, environmental strains produced more esterase, allowing them to degrade simple lipids (monoacylphospholipids) that can be present on hospital surfaces and/or in water, and are important to fungal nutrition. In summary, the phospholipase production profile of the different fungal groups was characterized as follow: $\mathrm{IA}<\mathrm{H}<\mathrm{RTMO}<\mathrm{RIC}$, whereas the esterase production was inversely proportional in the same fungal groups: $\mid A>H>R T M O>$ $\mathrm{RIC}$. Moreover, the isolates scraped from the foot interdigital space of immunocompetent patients (RIC) showed a significant difference between phospholipase and esterase production $(\mathrm{P}<0.05$, Student $t$-test; Figure 2E). Probably, these environmental strains may require an enzymatic system to degrade simple lipids such as monoacylphospholipids - esterase substrate - present in the environment. Meanwhile, further studies analyzing more Fusarium isolates from environmental and clinical material should be performed to confirm these hypotheses.

Phospholipases are ubiquitous enzymes with hydrolytic characteristics that attack phospholipids, mainly glycerophospolipids, common to all cell membranes, and involved in diverse processes such as membrane homeostasis, nutrient acquisition and generation of bioactive molecules. Some phospholipases play an important role in fungal pathogenesis and virulence (13). Ibrahim et al. (14) demonstrated that Candida albicans isolates with increased phospholipase activity were associated with a higher virulence profile in murine models of disseminated candidiasis. Ghannoum (6) showed that a phospholipase B1 knockout Candida strain produced fewer enzymes and was less virulent compared to a wild-type strain. In addition, phospholipase B produced by Paracoccidioides brasiliensis is also involved in the process of adhesion and internalization of yeast cells by MH-S cells and may enhance their virulence and the subsequent downregulation of macrophage activation (15).

In contrast to phospholipase, few studies have shown esterase production by fungi. Kumar et al. (11) described different Candida species isolated from immunocompromised patients, such as Candida albicans, C. tropicalis, C. lipolytica, and $C$. incospicua, which produced esterase,
Table 1. Phospholipase and esterase activities from 16 Fusarium spp isolated from hospital environment (IA), immunocompromised patients' blood cultures $(H)$, oot interdigital space scrapings from immunocompromised patients (RTMO),

\begin{tabular}{|c|c|c|c|c|c|}
\hline \multirow[t]{3}{*}{ Isolates } & \multirow[t]{3}{*}{ Species } & \multicolumn{4}{|c|}{ Pz values } \\
\hline & & \multicolumn{2}{|c|}{ Phospholipase activity } & \multicolumn{2}{|c|}{ Esterase activity } \\
\hline & & 15 days & 30 days & 15 days & 30 days \\
\hline IA-1 & FOSC & $0.92(+)$ & $0.88(+)$ & $0.77(+)$ & $0.30(+++)$ \\
\hline IA-2 & FSSC & $0.92(+)$ & $0.40(++)$ & $0.86(+)$ & $0.36(+++)$ \\
\hline IA-3 & FOSC & $0.90(+)$ & $0.79(+)$ & $0.81(+)$ & $0.37(+++)$ \\
\hline IA-4 & FSSC & $0.76(+)$ & $0.56(++)$ & $0.85(+)$ & $0.74(+)$ \\
\hline $\mathrm{H}-1$ & FSSC & $0.87(+)$ & $0.77(+)$ & $0.66(++)$ & $0.76(+)$ \\
\hline $\mathrm{H}-2$ & FSSC & $0.83(+)$ & $0.58(++)$ & $0.76(+)$ & $0.51(++)$ \\
\hline $\mathrm{H}-3$ & FSSC & $0.89(+)$ & $0.49(++)$ & $0.83(+)$ & $0.54(++)$ \\
\hline $\mathrm{H}-4$ & FSSC & $0.91(+)$ & $0.59(++)$ & $0.81(+)$ & $0.55(++)$ \\
\hline RTMO-1 & FSSC & $0.84(+)$ & $0.47(++)$ & $0.91(+)$ & $0.69(++)$ \\
\hline RTMO-2 & FSSC & $0.94(+)$ & $0.67(++)$ & $0.88(+)$ & $0.64(++)$ \\
\hline RTMO-3 & FSSC & $0.98(+)$ & $0.51(++)$ & $0.89(+)$ & $0.68(++)$ \\
\hline RTMO-4 & FSSC & $0.96(+)$ & $0.52(++)$ & $0.69(++)$ & $0.60(++)$ \\
\hline RIC-1 & FSSC & $0.83(+)$ & $0.46(++)$ & nd & $0.86(+)$ \\
\hline RIC-2 & FSSC & $0.84(+)$ & $0.43(++)$ & $0.80(+)$ & $0.93(+)$ \\
\hline RIC-3 & FSSC & $0.93(+)$ & $0.51(++)$ & $0.94(+)$ & $0.58(++)$ \\
\hline RIC-4 & FSSC & $0.86(+)$ & $0.54(++)$ & $0.82(+)$ & $0.58(++)$ \\
\hline
\end{tabular}

The isolates were incubated for 15 and 30 days on modified Sabouraud dextrose agar. The $\mathrm{Pz}$ value was scored into four categories: high $\mathrm{Pz}$ values of 1.0 were scored as negative enzymatic production, $\mathrm{Pz}$ values between 0.999 and 0.399 and 0.100 as high enzymatic production $(+++)$. FOSC $=$ Fusarium oxysporum species complex; FSSC = Fusarium solani species complex; nd = not determined.

and the increase in the level of $C$. albicans esterase activity was correlated with fungal growth (16). More recently, Palmeira et al. (12) reported that high levels of esterase activity in the fungus Fonsecaea pedrosoi were related to adherence to mammalian cells. Additionally, Malassezia furfur, an important etiologic agent of mycosis on the stratum corneum of human skin, produces high levels of extracellular esterase activity (17). Independently of the source of fungal isolation, all the strains analyzed in the present study showed resistance to amphotericin B, fluconazole and itraconazole in an in vitro antifungal susceptibility assay (data not shown).

Invasive fungal infections are serious and escalate health problems. They are associated with high morbidity and mortality (18). Current therapies are limited in safety and/or efficacy and resistant fungal pathogens are an emerging problem. Of the antifungal drugs marketed for systemic use, all, except echinocandins (which target the cell wall synthesis enzyme, $\beta-1,3$ glucan synthase) act on the fungal membrane lipid, ergosterol, or its biosynthetic pathways (19). Thus, new drugs acting on novel targets 
are needed. Virulence factors, like multifunctional enzyme phospholipase B1, are an example of potential targets for drug discovery (18).

Although some studies have shown the importance of Fusarium enzymatic hydrolysis in the production processes of dairy foods such as milk modification (20), our study is the first one to correlate the expression of the preferential hydrolytic enzyme fungal habitat. In this respect, the data obtained in the present study open several possibilities to study phospholipase and esterase activities by Fusarium

\section{References}

1. Nucci M, Anaissie E. Fusarium infections in immunocompromised patients. Clin Microbiol Rev 2007; 20: 695-704.

2. O'Donnell K, Sutton DA, Rinaldi MG, Sarver BA, Balajee $\mathrm{SA}$, Schroers $\mathrm{HJ}$, et al. Internet-accessible DNA sequence database for identifying fusaria from human and animal infections. J Clin Microbiol 2010; 48: 3708-3718.

3. Nelson PE, Dignani MC, Anaissie EJ. Taxonomy, biology, and clinical aspects of Fusarium species. Clin Microbiol Rev 1994; 7: 479-504.

4. Kratka J, Kovacikova E. The effect of temperature and age of strains of Fusarium oxysporum on its enzymatic activity. Zentralbl Bakteriol Naturwiss 1979; 134: 154-158.

5. Aktas E, Yigit N, Ayyildiz A. Esterase activity in various Candida species. J Int Med Res 2002; 30: 322-324.

6. Ghannoum MA. Potential role of phospholipases in virulence and fungal pathogenesis. Clin Microbiol Rev 2000; 13: 122143.

7. Scheel CM, Nucci M, Hurst SF, Park BJ, Balajee SA. Molecular typing of Fusarium isolates from a Brazilian hospital cluster indicates possible community-acquired infection (Abstract M-410). 40th Interscience Conference on Antimicrobial Agents and Chemotherapy. Toronto, September 17-20; 2000.

8. Price MF, Wilkinson ID, Gentry LO. Plate method for detection of phospholipase activity in Candida albicans. Sabouraudia 1982; 20: 7-14.

9. Schoofs A, Odds FC, Colebunders R, leven M, Goossens H. Use of specialised isolation media for recognition and identification of Candida dubliniensis isolates from HIV-infected patients. Eur J Clin Microbiol Infect Dis 1997; 16: 296-300.

10. Silva S, Negri M, Henriques M, Oliveira R, Williams DW, Azeredo J. Adherence and biofilm formation of non-Candida albicans Candida species. Trends Microbiol 2011; 19: 241 247.

11. Kumar CP, Menon T, Sundararajan T, Nalini S, Thirunarayan isolates, such as purification for better biochemical characterization of their activities, and their importance for biotechnology and during fungal-host interaction. In addition, these lipases may be considered to be an interesting target for antifungal drug discovery.

\section{Acknowledgments}

\author{
Research supported by CAPES, CNPq, and FAPERJ.
}

MA, Rajasekaran S, et al. Esterase activity of Candida species isolated from immunocompromised hosts. Rev Iberoam Micol 2006; 23: 101-103.

12. Palmeira VF, Kneipp LF, Alviano CS, dos Santos AL. Phospholipase and esterase production by clinical strains of Fonsecaea pedrosoi and their interactions with epithelial cells. Mycopathologia 2010; 170: 31-37.

13. Kohler GA, Brenot A, Haas-Stapleton E, Agabian N, Deva R, Nigam S. Phospholipase A2 and phospholipase B activities in fungi. Biochim Biophys Acta 2006; 1761: 1391-1399.

14. Ibrahim AS, Mirbod F, Filler SG, Banno Y, Cole GT, Kitajima $\mathrm{Y}$, et al. Evidence implicating phospholipase as a virulence factor of Candida albicans. Infect Immun 1995; 63: 19931998.

15. Soares DA, de Andrade RV, Silva SS, Bocca AL, Soares Felipe SM, Petrofeza S. Extracellular Paracoccidioides brasiliensis phospholipase B involvement in alveolar macrophage interaction. BMC Microbiol 2010; 10: 241.

16. Tsuboi R, Komatsuzaki H, Ogawa $H$. Induction of an extracellular esterase from Candida albicans and some of its properties. Infect Immun 1996; 64: 2936-2940.

17. Ran Y, Yoshiike T, Ogawa H. Lipase of Malassezia furfur: some properties and their relationship to cell growth. J Med Vet Mycol 1993; 31: 77-85.

18. Sorrell TC, Djordjevic JT, Chen SCA, Jolliffe KA. Fungal phospholipid metabolism for antifungal drug discovery. Microbiol Aust 2010; 31: 93-94.

19. Ostrosky-Zeichner L, Casadevall A, Galgiani JN, Odds FC, Rex JH. An insight into the antifungal pipeline: selected new molecules and beyond. Nat Rev Drug Discov 2010; 9: 719727.

20. Lilbaek HM, Fatum TM, Ipsen R, Sorensen NK. Modification of milk and whey surface properties by enzymatic hydrolysis of milk phospholipids. J Agric Food Chem 2007; 55: 29702978. 